
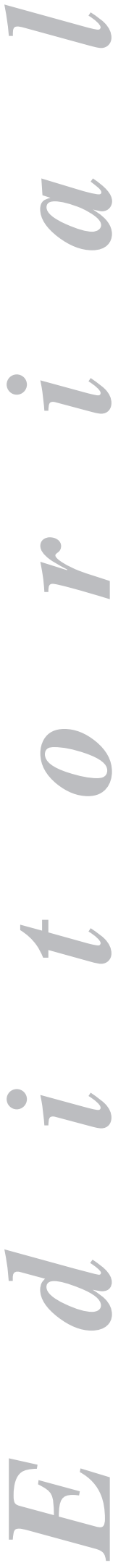

\section{Reexposición en hepatotoxicidad: ¿prueba de concepto o accidente terapéutico?}

Las reacciones hepatotóxicas se encuentran entre las reacciones adversas a medicamentos potencialmente más temibles -tanto por sus repercusiones económicas durante el proceso de desarrollo de los fármacos, como por su impacto en términos de morbimortalidad-y, por ello, vienen desafiando desde hace decenios a clínicos, farmacólogos, toxicólogos, industria farmacéutica y autoridades reguladoras. Para los gastroenterólogos y hepatólogos, la enfermedad hepática tóxica es además un problema desconcertante, debido al amplio número de sustancias capaces de inducir este tipo de reacciones, a la heterogeneidad de la presentación clínica y a la imposibilidad en el momento actual de establecer con certeza el diagnóstico en la inmensa mayoría de los casos. En este sentido, el primer elemento a considerar es que la lista de fármacos, drogas de abuso, plantas medicinales y suplementos dietéticos capaces de dañar el hígado crece de año en año (1) y así es prácticamente imposible descartar ninguna sustancia que el paciente esté tomando como potencial responsable de un cuadro hepático (2). En segundo término, y aunque la lesión hepatocelular aguda similar a la hepatitis vírica es la forma de presentación más común, la hepatotoxicidad es capaz de expresarse como cualquier otra enfermedad hepática aguda o crónica, incluyendo hepatitis crónica, cirrosis hepática o biliar, enfermedades vasculares y tumores. Ni siquiera los fármacos producen un patrón constante de lesión hepática (p. ej. amoxicilina-ácido clavulánico es considerado una hepatotoxina que induce cuadros colestásicos/mixtos, pero frecuentemente provoca también reacciones hepatocelulares) (3) y esta peculiaridad genera perplejidad en el clínico, erigiéndose en una dificultad añadida para la imputación diagnóstica.

Sin embargo, el principal obstáculo para la caracterización de las reacciones hepatotóxicas es la ausencia de marcadores clínicos, biológicos o anatomopatológicos específicos de toxicidad hepática. En las últimas décadas se han producido extraordinarios avances en la comprensión de la fisiopatología y en el diagnóstico de diversas enfermedades hepáticas, paralelos al desarrollo de la genómica, biología molecular y radiodiagnóstico. Si bien son también innegables los progresos habidos en la comprensión de la patogénesis de la lesión hepática tóxica producida por determinados compuestos (4), tales avances han tenido un impacto muy limitado en el reconocimiento de factores de susceptibilidad individual al efecto tóxico de los fármacos y en la disponibilidad de biomarcadores de hepatotoxicidad (5), especialmente en lo que se refiere a la reacciones hepatotóxicas impredecibles, por lo que el diagnóstico de esta variedad de enfermedad hepática continúa siendo en la actualidad muy rudimentario y se basa en la imputación de un medicamento u otro tóxico potencial por su relación temporal con el cuadro clínico y la pertinente exclusión de otras causas específicas de hepatopatía. Tal carencia ha tratado de suplirse con algunos instrumentos diagnósticos que integran toda la información disponible, para evaluar de 
forma probabilística la causalidad de un medicamento o tóxico, con el inconveniente de que no pueden ser comparados con un gold standard (6). Entre ellos, la escala diagnóstica de CIOMS/RUCAM ha demostrado ser la de mayor validez y reproducibilidad (7), pero es evidente que, con la gran cantidad de información clínica acumulada en los últimos años por algunos grupos cooperativos (8), hay un margen para el refinamiento de esta escala.

A pesar de la ausencia de un gold standard unánimemente aceptado para el diagnóstico, la respuesta positiva a una reexposición al agente sospechoso ha sido tradicionalmente considerada una evidencia robusta de la responsabilidad de un medicamento determinado en la aparición de una reacción adversa (9) y dicho concepto podría aplicarse también a la sospecha de reacciones hepatotóxicas. De hecho, las escalas diagnósticas fueron validadas utilizando casos con reexposición positiva $(10,11)$ y puntúan generosamente dicha eventualidad.

La reexposición dirigida arrastra, sin embargo, problemas éticos y prácticos. El eminente hepatotoxicólogo Hyman Zimmerman ya advirtió en su tratado sobre hepatotoxicidad (12) que una prueba de provocación en un caso de hepatotoxicidad, especialmente si la lesión hepática era del tipo hepatocelular con manifestaciones de hipersensibilidad -que incluyen fiebre, rash, eosinofilia y, con menor frecuencia, afectación concomitante de otros órganos tales como riñón o páncreas- aunque muy útil como prueba de concepto, ya que podría hacerse con una única dosis del medicamento, debido a la naturaleza alérgica del cuadro, podría tener consecuencias graves y estaría por ello contraindicada. En este contexto, la provocación originaría un incremento en la producción de neoantígenos, una situación en la cual la presencia de anticuerpos determinaría una intensa citólisis (13). Más recientemente, observaciones de carácter retrospectivo han sugerido que las reacciones hepatotóxicas con evidencia de alergia (eosinofilia periférica y hepática) tienen un curso más benigno que aquellas sin estas manifestaciones $(14,15)$. Así, y contrariamente al concepto popularizado por Zimmerman hace tres décadas (12), los conocimientos actuales sugieren que una prueba de provocación tendría mayor riesgo en las reacciones hepatocelulares sin síntomas de alergia asociados (denominadas metabólicas), dado que estudios recientes indican que dichas reacciones conllevan una mortalidad o necesidad de trasplante no inferior al $10 \%$ como predijo este autor $(8,16)$. Además, la exposición al agente responsable en estos últimos casos podría requerir un tratamiento de días o semanas para permitir la reaparición de los síntomas, lo cual es un grave inconveniente de orden práctico y, teniendo en cuenta que la positividad de la provocación no superaría el 40-60\% de los casos (12), un resultado negativo de la misma no excluiría el diagnóstico. En cualquier caso, es difícilmente justificable desde un punto de vista ético provocar una reacción con un fármaco que no sea considerado vital e irreemplazable, ya que el resultado no tendría entonces un valor más que puramente académico, a pesar de lo cual hay ejemplos en la literatura de este proceder (17-19). Únicamente cuando el fármaco responsable de un episodio de hepatitis se juzgue esencial y no haya alternativas terapéuticas evidentes, podría ensayarse una reexposición previo consentimiento informado del paciente (20).

Con mayor frecuencia, en la práctica clínica, la administración del agente responsable de una reacción hepatotóxica ocurre de forma involuntaria y accidental. En el presente número de la Revista Española de Enfermedades Digestivas Fernández-Castañer y cols. han revisado la frecuencia de la reexposición (en un $85 \%$ de los casos no intencionada) en una cohorte amplia de casos incluidos en el Registro Español de Hepatotoxicidad. Se trata del primer estudio en la literatura que analiza esta circunstancia y sus condicionantes de manera específica y la información que 
proporciona es relevante. Al margen de mostrar que la incidencia de reexposición en hepatotoxicidad no es excepcional, afectando al $6 \%$ de los casos, merece destacarse de los resultados de este estudio algunos hechos: a) la presencia de manifestaciones de hipersensibilidad fue más frecuente entre los casos con reexposición que en el global de pacientes incluidos en el Registro (39 vs. 23\%), lo que sugiere que la provocación de la reacción es más fácil si el mecanismo es alérgico, o bien que los casos de reexposición sin manifestaciones de hipersensibilidad podrían pasar desapercibidos con mayor frecuencia; b) la reexposición derivó en una evolución fatal o la necesidad de trasplante en más del $10 \%$ de los casos (4/31) y esta evolución desfavorable fue más frecuente en casos hepatocelulares; y c) las causas más frecuentes de reexposición accidental fueron la ausencia de diagnóstico claro del primer episodio de hepatotoxicidad, junto a circunstancias evitables como serían la falta de información sobre la reacción adversa inicial al paciente, al médico de cabecera y su constatación en el informe clínico. Asimismo, el episodio de reexposición fue debido en algunos casos a un fármaco distinto (reacción cruzada) (21). Estos últimos hallazgos subrayan la necesidad de mayor formación continuada a los médicos sobre reacciones adversas a fármacos, así como la importancia de extremar el celo en la comunicación sobre este tópico entre médicos de los distintos niveles asistenciales y de médicos a pacientes y de plasmar por escrito los diagnósticos de reacciones adversas a fármacos, aunque no hayan pasado de la mera sospecha clínica. Desafiando lo que sería común a las buenas prácticas jurídicas, un fármaco siempre será culpable de un incidente hepático del que sea sospechoso mientras no se demuestre lo contrario. Más vale destruir reputaciones que poner en el futuro de nuevo en juego la salud de aquellos pacientes que han tenido el infortunio de sufrir una alteración hepática al recibir un tratamiento farmacológico.

\author{
R. J. Andrade, M. Robles, N. Ortiz y M. I. Lucena ${ }^{1}$ \\ Unidad de Hepatología. Servicio de Aparato Digestivo. 'Servicio de Farmacología \\ Clínica. Hospital Universitario "Virgen de la Victoria". Málaga
}

\title{
Bibliografía
}

1. Kaplowitz N. Idiosyncratic drug hepatotoxicity. Nat Reviews Drug Discov 2005; 4: 489-99.

2. Schoepfer AM, Engel A, Fattinger K, Marbet UA, Criblez D, Reichen J, et al. "Herbal" does not mean "innocuous": 10 cases of severe hepatotoxicity associated with dietary supplements from Herbalife ${ }^{\circledast}$ products. J Hepatol 2007; 47: 521-6.

3. Lucena MI, Andrade RJ, Fernández MC, Pachkoria K, Peláez G, Durán JA, et al. Determinants of the clinical expression of amoxicillin-clavulanate hepatotoxicity: A prospective series from Spain. Hepatology 2006; 44: $850-6$.

4. Gunawan BK, Liu ZX, Han D, Hanawa N, Gaarde WA, Kaplowitz N. c-Jun N-terminal kinase plays a major role in murine acetaminophen hepatotoxicity. Gastroenterology 2006; 131: 165-78.

5. Davern TJ $2^{\text {nd }}$, James L, Hinson JA, Polson J, Larson AM, Fontana RJ, et al. Measurement of serum acetaminophen-protein adducts in patients with acute liver failure. Gastroenterology 2006; 130: 687-94.

6. Andrade RJ, Lucena MI. Drug-induced hepatotoxicity. N Engl J Med 2003; 349: 1974-6.

7. Lucena MI, Camargo R, Andrade RJ, Pérez-Sánchez C, Sánchez de la Cuesta F. Comparison of two clinical scales for causality assessment in hepatotoxicity. Hepatology 2001; 33: 123-30.

8. Andrade RJ, Lucena MI, Fernández MC, Peláez G, Pachkoria K, García-Ruiz E, et al. Drug-induced liver injury: An analysis of 461 incidences submitted to the Spanish Registry over a 10-year period. Gastroenterology 2005; 129: 512-21.

9. Edwards R, Aronson JK. Adverse drug reactions: Definitions, diagnosis and management. Lancet 2000; 356: $1255-9$.

10. Danan G, Bénichou C. Causality assessment of adverse reactions to drugs I. A novel method based on the conclusions of international consensus meetings: Application to drug-induced liver injuries. J Clin Epidemiol 1993; 46: 1323-30. 
11. Maria V, Victorino R. Development and validation of a clinical scale for the diagnosis of drug-induced hepatitis. Hepatology 1997; 26: 664-9.

12. Zimmerman HJ. Hepatotoxicity. The adverse effects of drugs and other chemicals on the liver. $2^{\text {nd }}$ ed. Philadelphia: Lippincott Williams \& Wilkins; 1999.

13. Dansette PM, Bonierbale E, Minoletti C, Beaune PH, Pessayre D, Mansuy D. Drug-induced immunotoxicity. Eur J Drug Metab Pharmacokinet 1998; 23: 443-51.

14. Björnsson E, Nordlinder H, Olsson R. Clinical characteristics and prognostic markers in disulfiram-induced liver injury. J Hepatol 2006; 44: 791-7.

15. Björnsson E, Kalaitzakis E, Olsson R. The impact of eosinophilia and hepatic necrosis on prognosis in patients with drug-induced liver injury. Aliment Pharmacol Ther 2007; 25: 1411-21.

16. Björnsson E, Olsson R. Outcome and prognostic markers in severe drug-induced liver disease. Hepatology 2005; 42: 481-9.

17. Marazuela M, Sánchez de Paco G, Jiménez I, Carraro R, Fernández-Herrera J, Pajares JM, et al. Acute pancreatitis, hepatic cholestasis, and erythema nodosum induced by carbimazole treatment for Graves' disease. Endocr J 2002; 49: 315-8.

18. Gershovich OE, Lyman AE Jr. Liver function test abnormalities and pruritus in a patient treated with atorvastatin: Case report and review of the literature. Pharmacotherapy 2004; 24: 150-4.

19. Philips BB, Digmann RR, Beck MG. Hepatitis associated with low dose venlafaxine for postmenopausal vasomotor symptoms. Ann Pharmacother 2006; 40: 323-7.

20. Andrade RJ, Robles M, Fernández-Castañer A, López-Ortega S, López-Vega MC, Lucena MI. Assessment of drug-induced hepatotoxicity in clinical practice: A challenge for gastroenterologists. World J Gastroenterol 2007; 13: 329-40

21. Fernández-Castañer A, García-Cortés M, Lucena MI, Borraz Y, Peláez G, Costa J, et al. Análisis de las causas, características y consecuencias de la reexposición al fármaco o compuesto responsable de un episodio de hepatotoxicidad. Rev Esp Enferm Dig 2008; 100 (5): 278-84. 